Cite this: Photochem. Photobiol. Sci., 2012, 11, 371

\title{
The eicosanoid response to high dose UVR exposure of individuals prone and resistant to sunburn
}

\author{
Anna Nicolaou, ${ }^{* a, b}$ Mojgan Masoodi, ${ }^{a}$ Karl Gledhill, ${ }^{b}$ Ann Katarina Haylett, ${ }^{c}$ Anthony John Thody, ${ }^{d}$ \\ Desmond John Tobin ${ }^{b}$ and Lesley Elizabeth Rhodes ${ }^{c}$
}

Received 27th August 2011, Accepted 3rd November 2011

DOI: 10.1039/c1pp05272a

High personal UVR doses can be gained during leisure activities, causing intense self-resolving inflammation (sunburn) of unprotected skin. UVR activates release of membrane fatty acids and upregulates their metabolism by cyclooxygenases (COX) and lipoxygenases (LOX) to different eicosanoids. While COX-derived prostaglandin $(\mathrm{PG}) \mathrm{E}_{2}$ is a potent mediator of sunburn vasodilatation, LOX-derived 15-hydroxyeicosatetraenoic acid (HETE) and its lipoxin metabolites may contribute to sunburn limitation. We explored the relationships between expression of these lipid mediators and the clinical and histological outcomes, comparing responses of individuals prone and more resistant to sunburn. An acute UVR exposure of 12 SED (standard erythema dose) was applied to buttock skin of 32 white Caucasians ( $\mathrm{n}=16$ phototype I/II, $\mathrm{n}=16$ phototype III/IV), and over the subsequent $72 \mathrm{~h}$ assessments were made of skin erythema, immunohistochemical expression of leukocyte markers, COX-2, 12-LOX, 15-LOX and nitric oxide synthase (NOS), and eicosanoid levels by LC/ESI-MS/MS. Evidence of a significant inflammatory response was seen earlier in phototype I/II with regard to expression of erythema ( $4 \mathrm{~h}, p<0.001)$, neutrophil infiltration $(24 \mathrm{~h}, p=0.01)$, epidermal COX-2 (24 h, $p<0.05)$ and 12-LOX (24 h, $p<0.01)$, and dermal eNOS $(24 \mathrm{~h}, p<0.05)$ proteins, although CD3+ lymphocyte infiltration showed an earlier increase in phototype III/IV ( $24 \mathrm{~h}, p<0.05)$. Although erythema was equivalent at $72 \mathrm{~h}$ in both groups, phototype I/II showed higher $\mathrm{PGE}_{2}$ accompanied by elevated 15-HETE, and a strong positive correlation was seen between these mediators $(\mathrm{n}=18, r=$ $0.805, p=0.0001)$. Hence anti-inflammatory eicosanoid 15 -HETE may temper the pro-inflammatory milieu in sunburn, having greater influence in those prone to sunburn than those more resistant, given the same high UVR exposure conditions.

\section{Introduction}

Excessive exposure to sunlight can cause a range of cutaneous health problems, particularly in fair-skinned people. Solar ultraviolet radiation (UVR) is the major environmental factor contributing to skin carcinogenesis, and while both UVA (320$400 \mathrm{~nm})$ and UVB (290-320 nm) contribute to acute and longer term damage, UVB is the principal waveband inducing the acute inflammatory response that is sunburn, and is believed to play a larger role in skin cancer development. ${ }^{1}$ A clinical classification of four sun-reactive skin types (phototypes) in fair-skinned people

${ }^{a}$ Bradford School of Pharmacy, School of Life Sciences, University of Bradford, Richmond Road, Bradford, UK, BD71DP. E-mail: a.nicolaou@ bradford.ac.uk; Fax: +44 1274 235600; Tel: +44 1274234717

${ }^{b}$ Centre for Skin Sciences, School of Life Sciences, University of Bradford, Bradford, UK

'Photobiology Unit, Dermatological Sciences, School of Translational Medicine, University of Manchester, Manchester Academic Health Science Centre, Salford Royal NHS Foundation Hospital, Manchester, UK

'Institute of Cellular Medicine, Dermatological Sciences, Newcastle University, Newcastle upon Tyne, UK was developed based on an individual's susceptibility to sunburn and ability to $\tan ^{2}$ Broadly, these can be divided into those who sunburn easily and tan with difficulty (phototype I/II) and those who tan readily and are less susceptible to sunburn (phototype III/IV); a higher incidence of melanoma and non-melanoma skin cancer is seen in phototype I/II. ${ }^{1}$

Sunburn inflammation is characterised clinically by erythema, as a consequence of cutaneous vasodilatation, and histologically by the dermal infiltration of neutrophils and lymphocytes, and the presence of apoptotic keratinocytes. Prostaglandins (PG), nitric oxide (NO) and pro-inflammatory cytokines are soluble mediators underlying some of these changes. ${ }^{3-9}$ Recently, it has become apparent that a wider range of eicosanoids are involved in sunburn inflammation. ${ }^{10}$ The skin demonstrates intense metabolism of polyunsaturated fatty acids (PUFA), and an array of lipid mediators are produced via cutaneous isoforms of cyclooxygenase (COX), lipoxygenase (LOX) and cytochrome P450 (CYP) oxygenases. ${ }^{11-13}$ The omega-6 (n-6) PUFA, arachidonic acid, is metabolised via COX-1/-2 to, predominantly, $\mathrm{PGE}_{2}, \mathrm{PGF}_{2 \mathrm{a}}$ and $\mathrm{PGD}_{2}$, via 12-LOX to 12-hydroxyeicosatetraenoic (HETE) acid, 
through 15-LOX to 15-HETE, and via uncertain pathways to 8HETE and 11-HETE. At low concentrations eicosanoids modulate physiological processes. However, following an inflammatory stimulus such as UVR, production of $\mathrm{PGE}_{2}$ and 12-HETE is upregulated and whilst these lipids elicit inflammatory, immunomodulatory and chemotactic reactions, their impact could be tempered by UVR-upregulation of anti-inflammatory mediators. In this context, the UVR- upregulation of 15-HETE is of particular interest: 15-HETE has been shown to inhibit 12-HETE in human keratinocytes and skin sections, ${ }^{14,15}$ whilst it is a precursor to the anti-inflammatory lipoxins. ${ }^{16}$ Furthermore, $\mathrm{PGE}_{2}$ can induce 15-LOX expression thus modulating the levels of 15-HETE and, potentially, lipoxins. ${ }^{17}$ These observations suggest interplay between cutaneous pro- and anti-inflammatory eicosanoids in sunburn meriting further investigation.

There is cross-talk between the COX and nitric oxide synthase (NOS) pathways and PG/NO production, ${ }^{18,19}$ such that the two mediators may work in synergy to mediate sunburn vasodilatation. ${ }^{5}$ Increased NO production following UVR exposure may also contribute to melanogenesis and immunosuppression. ${ }^{20}$ Production of $\mathrm{NO}$ is mediated via the different isoforms of NOS expressed in cutaneous cells, i.e. keratinocytes constitutively express the neuronal isoform (nNOS), melanocytes in the basal layer of the epidermis express eNOS and nNOS, fibroblasts express the endothelial isoform (eNOS), whilst expression of the inducible isoform (iNOS) can be stimulated in a range of skin cells..$^{20,21}$

The objective of this study was to examine the relationships between cutaneous pro- and anti-inflammatory lipid production and accompanying inflammatory outcomes. We aimed to compare patterns seen in phototypes I/II and III/IV under conditions of high standardised (12 standard eythema doses, SED) UVR exposure on unprotected skin, as people do not necessarily limit their sun-exposure according to their propensity to burn. Exposure to such a high UVR dose may occur during outdoor activities including cycling, sailing and sunbathing. ${ }^{22-24} \mathrm{~A}$ detailed lipidomics assessment was performed over a $72 \mathrm{~h}$ period postUVR, with focus on vasodilatory prostanoids (particularly $\mathrm{PGE}_{2}$ ), leucocyte chemoattractants (particularly 8-, 11- and 12-HETE) and anti-inflammatory lipids (particularly 15-HETE), alongside expression of COX, LOX and NOS proteins, and clinical and histological inflammation.

\section{Materials and methods}

\subsection{Subjects}

A total of 32 healthy adult white Caucasian subjects were recruited for this study ( 20 females, 12 males; mean age 38 years, range 19-60 years). These comprised 16 subjects with a history of burning easily and tanning poorly following sun exposure (phototype I/II) and 16 subjects with a history of tanning easily and more resistance to burning (phototype III/IV). Specifically, volunteers were asked the question, "How does your skin typically react after approximately 30 min exposure to midday sun on the first sunny summer's day when you expose your skin", with follow up questions for clarification of sunburn and suntan tendency according to Fitzpatrick. ${ }^{2}$ Only individuals with clear responses placing them into I/II or III/IV were recruited to the study. Exclusions from the study were: sunbathing or sunbed use in the previous 3 months; use of photosensitizing or anti-inflammatory medication; history of skin cancer or photosensitivity disorders. The study conformed to the Declaration of Helsinki 2000, approval was obtained from North Manchester Local Research Ethics Committee and all volunteers gave written informed consent.

\subsection{UV irradiation}

The UV irradiation source used was a fluorescent UVB lamp, (Waldmann UV6, emission 290-400 nm, peak $313 \mathrm{~nm}$, Herbert Waldmann GmbH, Villingen-Schwenningen, Germany). Lamp output was measured before each irradiation using a calibrated sensor (Waldmann UV meter, Herbert Waldmann GmbH, Villingen-Schwenningen, Germany) traceable to UK National Physical Laboratory standards, and the UVR doses applied were kept constant by adjusting the length of irradiation. Individual sunburn threshold (minimal erythema dose, MED) was determined to confirm the differential burning tendency of the two volunteer groups. A geometric series of UVR doses ranging from 13 to $128 \mathrm{~mJ} \mathrm{~cm}^{2}$ of erythemally weighted UVR was applied in a horizontal row to the upper buttock skin. The MED was assessed at $24 \mathrm{~h}$ and defined as the lowest dose of UVR that resulted in visually detectable erythema. Volunteers were given a standardised high-dose UVR challenge of 12 SED, where 1 SED is equal to $100 \mathrm{~J} \mathrm{~m}^{-2}$ of erythemal UVR (approximately $4 \times$ MED for skin type II). ${ }^{24}$ This dose may be gained on unprotected skin during outdoor activities, including during the summer months in Europe and on beach holidays. ${ }^{22,23,25}$ This was utilised for the purposes of erythema time course determination ( $\mathrm{n}=32$ subjects), suction blister fluid sampling for lipidomic analysis $(\mathrm{n}=18$ subjects) or skin punch biopsy sampling for immunohistochemical investigation $(\mathrm{n}=12$ subjects).

\subsection{Assessment of erythemal responses}

Erythemal responses were quantified using a reflectance instrument (Diastron, Andover, UK) which gives an erythema index related to the blood content of the superficial dermis. Triplicate measurements were taken from the test sites and adjacent unirradiated skin, and the means calculated. UVR erythema dose response modelling was performed using a dedicated data analysis package (Regional Medical Physics Department, Gateshead \& Tyneside Authority, UK) to ascertain an individual's D30, i.e. the dose increasing the skin's erythema index by 30 units compared with unirradiated skin. The objective threshold value approximates the individual's visually assessed MED. ${ }^{26}$

\subsection{Suction blister sampling}

Suction blister fluid was sampled from unirradiated skin $(0 \mathrm{~h})$ and from skin at 24 and $72 \mathrm{~h}$ following exposure 12 SED of UVR. Each volunteer provided a sample from an unirradiated site on one buttock, and samples from two irradiated sites on the contralateral buttock, each site being sampled on one occasion. Suction blister cups with $1 \mathrm{~cm}$ central aperture were applied with a vacuum pressure of $250 \mathrm{~mm} \mathrm{Hg}$ as previously described. ${ }^{27}$ When blisters had formed (after approximately $90 \mathrm{~min}$ ) the fluid was aspirated with a 23 gauge needle, snap frozen and stored at $-80^{\circ} \mathrm{C}$ awaiting analysis. 


\subsection{Lipidomic analysis}

Lipidomic analyses were performed as described by Masoodi et al. ${ }^{28,29}$ Briefly, blister fluid samples (typical volumes 50-200 $\mu \mathrm{l}$ ) were diluted with methanol-water $(15 \% \mathrm{w} / \mathrm{w})$ to a final volume of $3 \mathrm{ml}$. Internal standards (40 ng $\mathrm{PGB}_{2}-d 4$ and $80 \mathrm{ng}$ 12-HETEd8) (Cayman Chemicals, Ann Arbor, MI, USA) were added to each sample. The resultant solutions were acidified to $\mathrm{pH} 3$ and immediately applied to pre-conditioned solid phase extraction cartidges (C18-E, Phenomenex, Macclesfield UK) to extract the lipid mediators. Chromatographic analysis was performed on a C18 column (Luna $5 \mu$, Phenomenex) using a Waters Alliance 2695 HPLC pump coupled to an electrospray (ESI) triple quadrupole Quattro Ultima mass spectrometer (Waters, Elstree, Hertsfordshire, UK). Instrument control and data acquisition were performed using MassLynx ${ }^{\mathrm{TM}}$ V4.0 software. The following multiple reaction monitoring (MRM) transitions were used for the assay of prostanoids, hydroxy fatty acids and other lipid mediators: $\mathrm{PGE}_{1} m / z 353>317 ; \mathrm{PGE}_{2} m / z 351>271 ; \mathrm{PGF}_{2 \alpha} \mathrm{m} / z 353>$ 193; 8-HETE $m / z 319>155$; 11 -HETE $m / z 319>167$; 12 HETE $m / z 319>179$; 15-HETE $m / z 319>175$; 13-HODE $m / z 295>195$. Results are expressed as pg metabolite/mg of protein based on calibration lines constructed with commercially available eicosanoid standards (Cayman Chemicals, Ann Arbor, USA). Protein content was estimated using the BioRad protein assay kit with BSA as standard (BioRad, Hemel Hempstead, UK).

\subsection{Immunohistochemical assessment}

Skin punch biopsies ( $5 \mathrm{~mm}$ diameter) were sampled from unirradiated skin $(0 \mathrm{~h})$ and irradiated skin at 4,24 and $72 \mathrm{~h}$ following $12 \mathrm{SED}$ of UVR. Biopsies were snap-frozen and stored at $-80{ }^{\circ} \mathrm{C}$ prior to immunohistochemical analysis. Frozen sections $(6 \mu \mathrm{m})$ were air-dried before fixation in ice-cold acetone (Fisher Scientific UK Ltd., Loughborough, UK) for $10 \mathrm{~min}$. Endogenous peroxidase was quenched in hydrated tissue sections with $0.5 \% \mathrm{H}_{2} \mathrm{O}_{2}$ (Sigma-Aldrich Co. Ltd, Gillingham, UK) before blocking with 10\% goat serum (Sigma-Aldrich) for $1 \mathrm{~h}$. Primary antibody was added to the sections at the required dilution and incubated overnight at $4{ }^{\circ} \mathrm{C}$. The primary antibodies used in this study were as follows. Neutrophil elastase: MAB 1056, dilution 1:70 (Chemicon Europe Ltd, Chandlers Ford, UK); $\mathrm{CD}^{+}$: NCL-CD3, dilution $1: 40$ (NovoCastra ${ }^{\mathrm{TM}}$ Reagents, Leica Biosystems Ltd, Newcastle upon Tyne, UK); 12-LOX: ab23678, dilution $1: 200$ (Abcam plc, Cambridge, UK); 15-LOX: 10004454, dilution $1: 100$ (Cayman Chemical, Boldon, UK); COX-1: 210710-1, dilution $1: 100$ (Alexis Biochemicals, Lausen, Switzerland); COX-2: ALX-210-712-1, dilution 1:100 (Alexis Biochemicals, Lausen, Switzerland); nNOS: SC-648, dilution 1:100 (Santa Cruz Biotechnology, CA, USA); iNOS: SC-651, dilution 1: 100 (Santa Cruz Biotechnology, CA, USA); eNOS: SC-653, dilution 1:100 (Santa Cruz Biotechnology, CA, USA). After washing in PBS, skin sections were incubated with biotinylated antimouse secondary antibody (DakoCytomation Ltd, Ely, UK) for $30 \mathrm{~min}$, washed in PBS, and incubated with streptavidin peroxidase (DakoCytomation) for $30 \mathrm{~min}$. Sections were developed using amino-ethylcarbazole chromogen (Vector Laboratories Ltd, Peterborough, UK) for 5-10 min, washed in distilled water, lightly counterstained in Meyers Haematoxylin (Sigma-Aldrich) with "blueing" in Scott's tap water, and mounted in glycergel mounting medium (DakoCytomation). The sections were assessed using a Nikon Eclipse $80 \mathrm{i}$ microscope at $\times 200$ original magnification and a Nikon Digital Sight DS-U1 camera (Nikon UK Ltd., Kingston upon Thames, UK).

\subsection{Assessment of immunohistochemical staining}

Slides were blinded prior to assessment. Neutrophils and CD3 ${ }^{+}$ cells were counted in 3 randomly selected epidermal and dermal high power fields (hpf) at $\times 400$ original magnification and mean cell number was calculated. This technique was employed for each individual at each of the 4 time points. COX-1,-2/12-, $15-\mathrm{LOX} / \mathrm{nNOS}$, iNOS, eNOS staining was quantified in terms of relative staining intensity, according to the following scoring system: $0=$ no staining, $1=$ weak staining, 2 = moderate staining, 3 = intense staining. 12-LOX, 15-LOX, COX-1 and COX-2 were graded in epidermal and dermal hpf at $\times 200$ original magnification. nNOS, iNOS, eNOS were graded in suprabasal and basal epidermal and in dermal hpf at $\times 200$ original magnification due to the clear differences in staining patterns for these enzymes at these locations. Assessment was made for each individual at each of the 4 time points.

\subsection{Statistical analysis}

Data were analysed with StatsDirect and PASW Statistics 18. Normal distribution was assessed by the Shapiro-Wilks test. Nonparametric data were analysed using the Kruskal-Wallis test with adjustment for multiple comparisons. Parametric data were analysed using unpaired $t$-test or one-way ANOVA with Bonferroni's correction for multiple comparisons, as appropriate. Correlations between levels were examined by the Pearson Correlation test. All results are compared with levels in un-irradiated tissue unless otherwise stated. Statistical significance was accepted at the $p<$ 0.05 level.

\section{Results}

\subsection{MED testing}

Since the criteria used for assigning skin phototype are subjective, ${ }^{2}$ direct testing of each individual's sunburn threshold, i.e. MED to UVR was performed. As shown in Fig. 1A a statistically significant difference ( $p=0.037$, unpaired $t$-test) was noted between the skin type I/II and III/IV individuals, indicating the two groups were distinct in their erythemal response to UVR.

\subsection{UV-erythema dose response}

A UV-erythema dose response was also plotted from the measured erythema index of the geometric series of UV doses $(n=18)$; from this a further, objective, threshold dose for erythema, i.e. the D30 which approximates the visually assessed MED, was calculated. This also showed a statistically significant difference ( $p=0.02$, unpaired $t$-test) between the two skin type individuals (Fig. 1B). 
A. MED

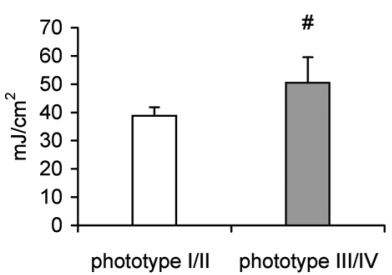

B. UV threshold Dose

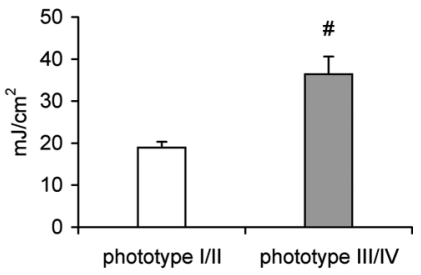

C. erythema index

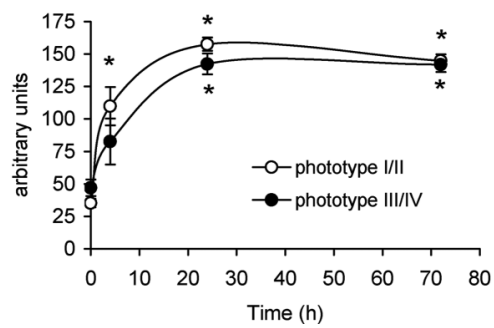

Fig. 1 Minimal erythema dose (MED) (A), UV threshold dose (B), and time course of the erythemal response (erythema index) following exposure to 12 SED of UVR (C), in subjects of phototypes I/II and III/IV. Results are expressed as mean \pm SEM ( $\mathrm{n}=16$ subjects per phototype group). \# $p<0.05$ comparing phototype I/II to III/IV; $* p<0.001$ comparing all data to un-irradiated skin $(0 \mathrm{~h})$.

\subsection{Time course of the erythema response in phototypes I/II and III/IV}

All subjects received a standardised dose of 12 SED of UVR in order to compare responses of the phototypes to the same intense UVR dose that could be gained by unprotected skin during leisure activities. Both subject groups showed a peak erythema at $24 \mathrm{~h}$ and erythema was maintained at $72 \mathrm{~h}$ post irradiation (Fig. 1C), with no statistically significant differences when comparing the two groups. However, a greater clinical inflammatory response

\section{A. neutrophils in dermis}
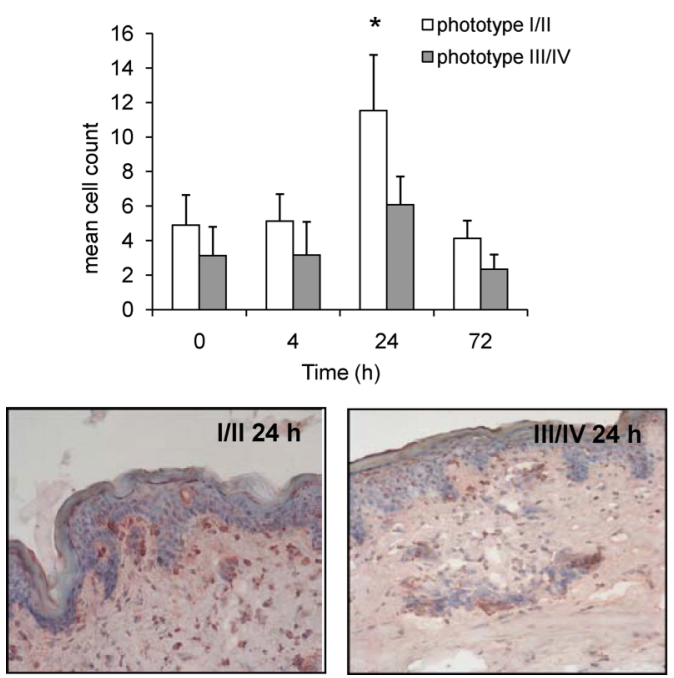

was apparent in phototype I/II at the earliest time point, with a statistically significant elevation of the erythema index in phototype I/II after $4 \mathrm{~h}(p<0.001)$ whilst in phototype III/IV the erythema index became statistically significantly different from baseline at $24 \mathrm{~h}$ post UVR $(p<0.001)$. The erythema index of the 2 groups was equivalent at $72 \mathrm{~h}$.

\subsection{Infiltration of neutrophils and CD3+ lymphocytes post UVR}

Dermal infiltrations of neutrophils and CD3+ lymphocytes were observed post UVR (Fig. 2A, 2B). In skin phototype I/II the neutrophil infiltration reached its peak at $24 \mathrm{~h}$ post UVR ( $p=$ 0.01 ); in phototype III/IV this also appeared to peak at $24 \mathrm{~h}$ but did not reach statistical significance. In skin type I/II the CD3+ cell infiltration peaked at $72 \mathrm{~h}$ post $\operatorname{UVR}(p=0.009)$, while in skin type III/IV a statistically significant increase was observed at the earlier time point of $24 \mathrm{~h}(p=0.014)$ and persisted at $72 \mathrm{~h}$ $(p=0.043)$ post UVR.

\subsection{Expression of cyclooxygenase-1 and -2 post UVR}

Cyclooxygenase expression was assessed in the epidermis and dermis of skin punch biopsies. The expression of constitutive COX-1 in non-irradiated skin $(0 \mathrm{~h})$ of phototypes I/II and III/IV was similar (Fig. 3A, 3B). COX-1 expression increased in the epidermis and dermis of phototype III/IV at, respectively, $72 \mathrm{~h}$ $(p=0.006)$ and $24 \mathrm{~h}(p=0.013)$ post UVR. Conversely, there was no statistically significant change in the expression of COX-1 in phototype I/II, in epidermis or dermis.

We did not detect inducible COX-2 in the epidermis of unirradiated $(0 \mathrm{~h})$ phototype I/II although it was present in phototype III/IV (Fig. 3C). UVR upregulated COX-2 in the epidermis and dermis of skin phototype I/II, its expression peaking at $24 \mathrm{~h}$ ( $p=$ 0.019 and $p=0.032$, respectively) post UVR. COX-2 expression did not significantly alter after UVR in phototype III/IV (Fig. 3C, 3D).

\section{B. CD3+ cells in dermis}
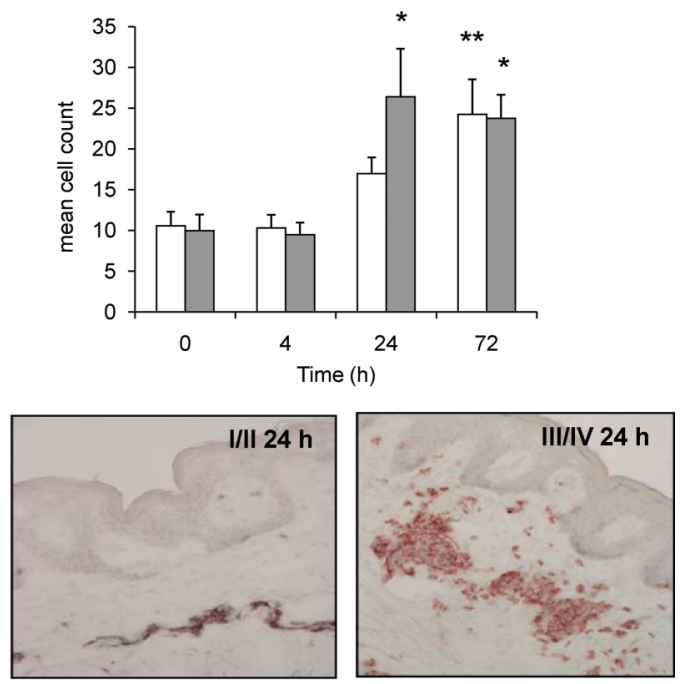

Fig. 2 Immunohistochemical assessment of neutrophil (A) and CD3+ lymphocytic (B) infiltrate in dermis of unirradiated (0 h) and irradiated (4, 24 and $72 \mathrm{~h}$ ) human skin phototype I/II and III/IV, following 12 SED of UVR. Representative photomicrograms show cell infiltration at $24 \mathrm{~h}$ post UVR, in both skin phototypes. Results are expressed as mean \pm SEM ( $\mathrm{n}=6$ subjects per phototype group). ${ }^{*} p<0.05,{ }^{* *} p<0.01$ comparing data to un-irradiated skin $(0 \mathrm{~h})$. 


\section{A. COX-1 in epidermis}

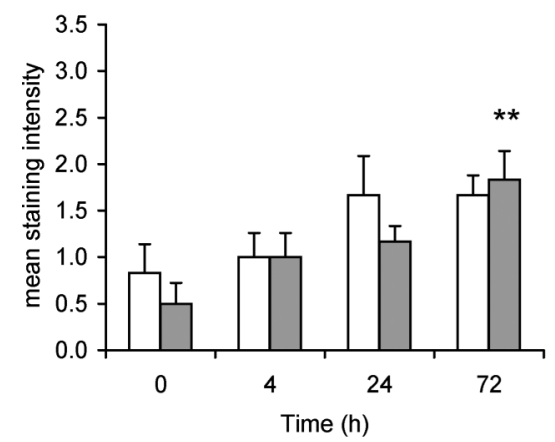

C. COX-2 in epidermis

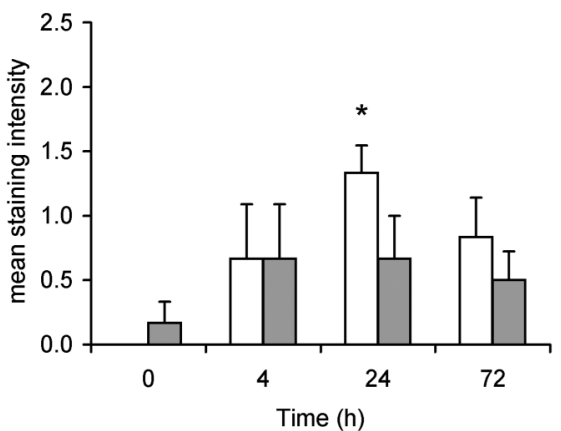

$\square$ phototype I/II

B. COX-1 in dermis

口phototype III/IV

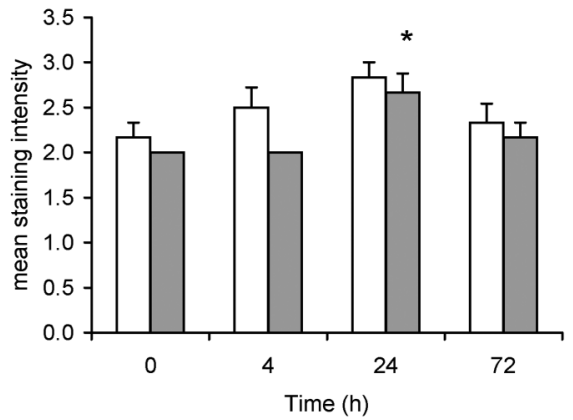

D. COX-2 in dermis

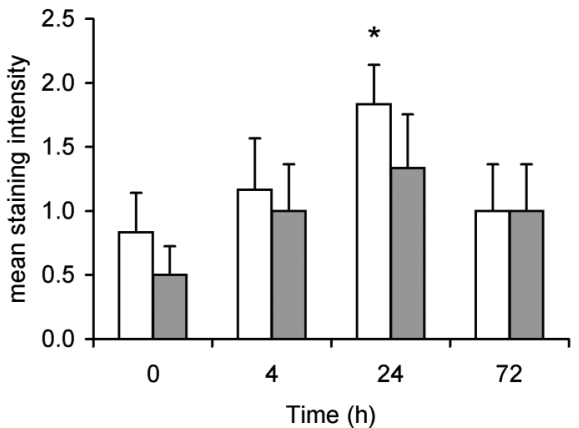

Fig. 3 Immunohistochemical assessment of COX-1 and COX-2 expression in skin punch biopsies of unirradiated (0 h) and irradiated (4, 24 and $72 \mathrm{~h})$ human skin phototype I/II and III/IV, following 12 SED of UVR. Expression of COX-1 in epidermis (A) and dermis (B); expression of COX-2 in epidermis (C) and dermis (D). Results are expressed as mean \pm SEM ( $\mathrm{n}=6$ subjects per phototype group). ${ }^{*} p<0.05,{ }^{* *} p<0.01$ comparing data to un-irradiated skin $(0 \mathrm{~h})$.

\subsection{Expression of 12- and 15-lipoxygenase post UVR}

The expression of 12- and 15-LOX was also assessed in the epidermis and dermis of skin punch biopsies. Epidermal expression of 12-LOX was upregulated following UVR and reached statistical significance for phototype I/II at $24 \mathrm{~h}(p=0.002)$ and $72 \mathrm{~h}(p=$ 0.007 ; Fig. 4A). A similar trend was noted for phenotype III/IV, but the increase did not reach statistical significance. In phototype I/II, dermal 12-LOX expression increased at $4 \mathrm{~h}(p=0.016)$ and $24 \mathrm{~h}(p=0.005)$ post UVR, but there was no difference in the expression of this protein in phototype III/IV (Fig. 4B).

Epidermal expression of 15-LOX was similar in both phototype groups (Fig. 4C) and, although appeared upregulated post UVR, the increase did not reach statistical significance. However, dermal 15-LOX expression increased in both phototype groups, peaking at $24 \mathrm{~h}$ post UVR ( $p=0.026$ for phototype I/II and $p=0.001$ for phototype III/IV) (Fig. 4D).

\subsection{Expression of nitric oxide synthase post UVR}

The expression of three isoforms of NOS, namely nNOS (constitutive, neuronal type), iNOS (inducible) and eNOS (endothelial cell type) was assessed in the epidermis and dermis of skin punch biopsies.

Neuronal NOS was detected in the basal layer of the epidermis and in the dermis of unirradiated skin $(0 \mathrm{~h})$ in both phototype groups (Fig. 5B, 5C). Its expression increased in the suprabasal level of the epidermis post UVR (Fig. 5A), but not in the basal
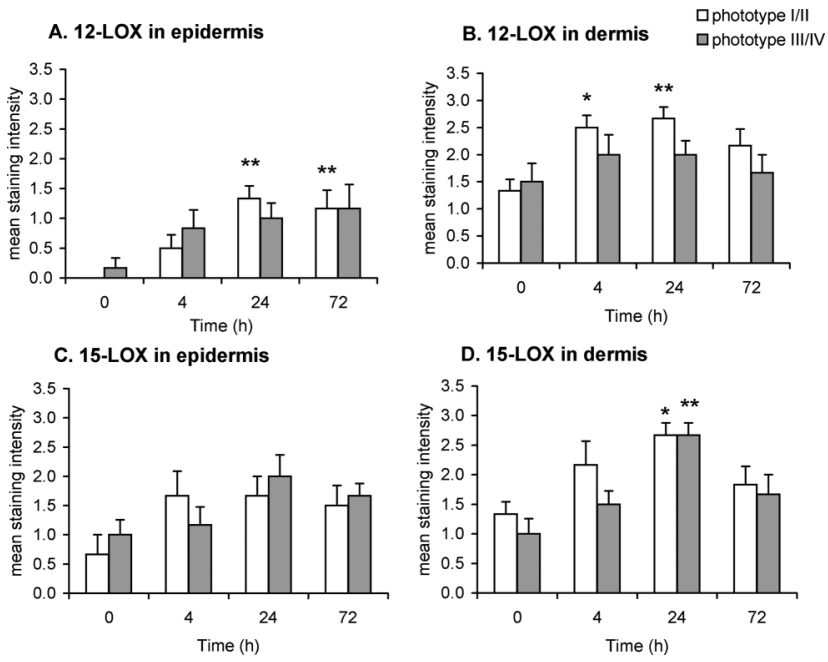

Fig. 4 Immunohistochemical assessment of 12-LOX and 15-LOX expression in skin punch biopsies of unirradiated $(0 \mathrm{~h})$ and irradiated $(4,24$ and $72 \mathrm{~h}$ ) human skin phototype I/II and III/IV, following 12 SED of UVR. Expression of 12-LOX in epidermis (A) and dermis (B); expression of 15-LOX in epidermis (C) and dermis (D). Results are expressed as mean \pm SEM ( $\mathrm{n}=6$ subjects per phototype group). ${ }^{*} p<0.05,{ }^{* *} p<0.01$ comparing data to un-irradiated skin $(0 \mathrm{~h})$.

layer. In phototype I/II, dermal expression of nNOS increased $24 \mathrm{~h}$ post UVR $(p=0.034)$ whilst no such significant change was noted in phototype III/IV. 


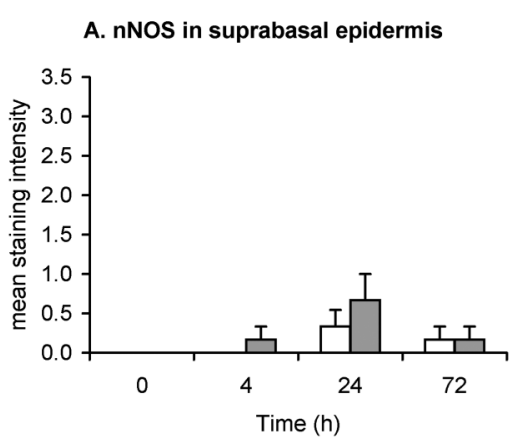

D. iNOS in suprabasal epidermis

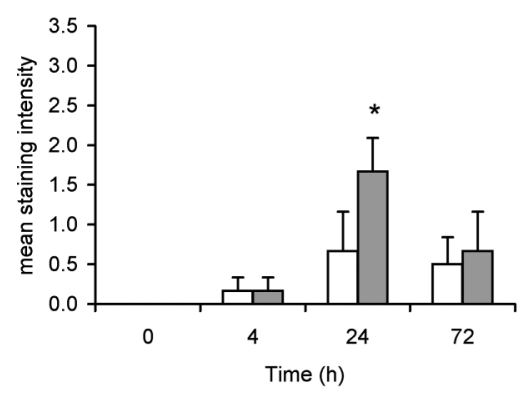

G. eNOS in suprabasal epidermis

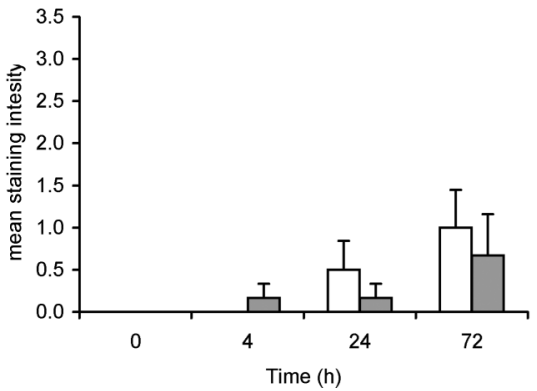

B. $n N O S$ in basal epidermis

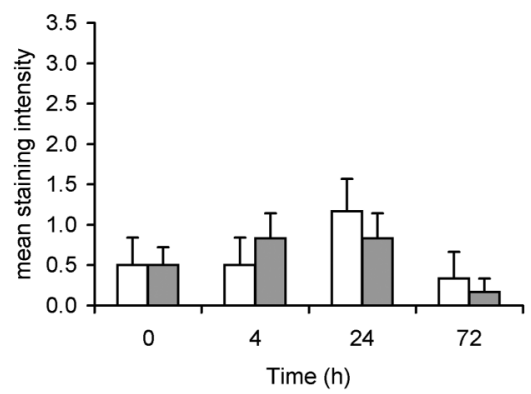

E. iNOS in basal epidermis

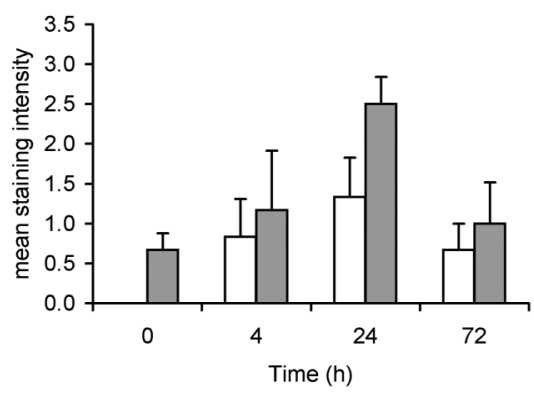

H. eNOS in basal epidermis

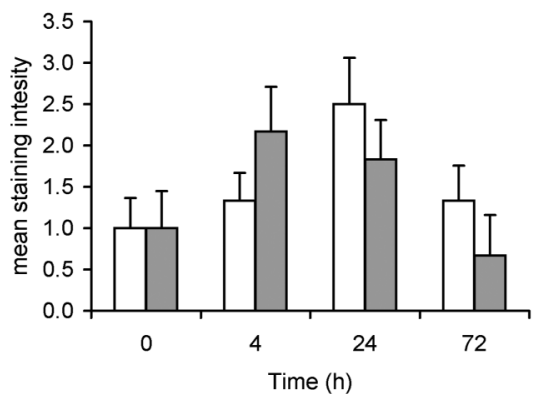

$\square$ phototype I/II 口phototype III/IV

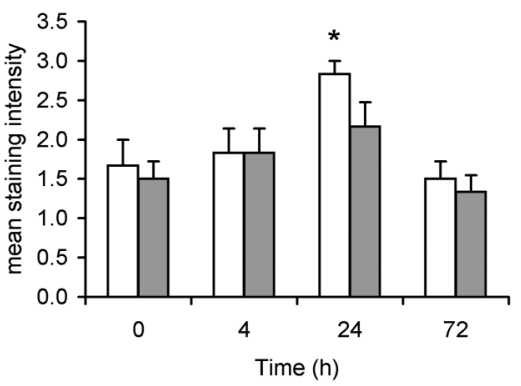

F. iNOS in dermis

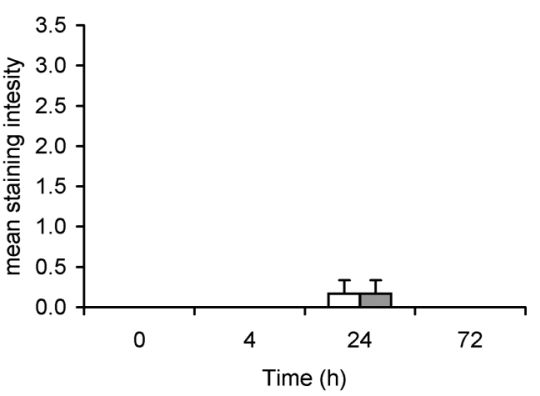

I. eNOS in dermis

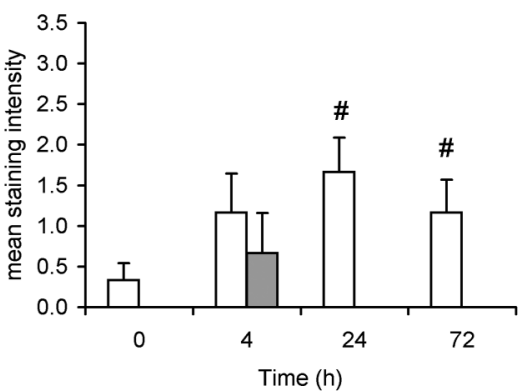

Fig. 5 Immunohistochemical assessment of neuronal, inducible and endothelial NOS (nNOS, iNOS and eNOS respectively) expression in skin punch biopsies of unirradiated $(0 \mathrm{~h})$ and irradiated $(4,24$ and $72 \mathrm{~h}$ ) human skin phototype I/II and III/IV, following 12 SED of UVR. Expression of nNOS in suprabasal (A) and basal (B) epidermis and dermis (C); expression of eNOS in suprabasal (D) and basal (E) epidermis and dermis (F); expression of eNOS in suprabasal (G) and basal (H) epidermis and dermis (I). Results are expressed as mean \pm SEM ( $\mathrm{n}=6$ subjects per skin phototype group). ${ }^{*} p<$ 0.05 comparing data to un-irradiated skin $(0 \mathrm{~h})$ and $\# p<0.05$ comparing skin phototype I/II to III/IV.

Inducible NOS was not detected in unirradiated skin in either phototype (Fig. 5E, 5F), except in the basal epidermis of skin phototype III/IV (Fig. 5E). UVR upregulated the expression of iNOS in the suprabasal layer of the epidermis in phototype III/IV $(p=0.013)$. Finally, some expression of iNOS was noted $24 \mathrm{~h}$ post UVR in the dermis of both skin types (Fig. 5F).

Endothelial NOS was present in the basal epidermis of both phototypes pre UVR exposure (Fig. 5G, 5H). UVR did not have any statistically significant effect in the expression of eNOS in the epidermis of either phototype, although a trend towards increased expression was noted (Fig. 5H). In contrast, dermal eNOS was detected mainly in skin phototype I/II (Fig. 5H). Skin types I/II and III/IV differed in terms of dermal eNOS expression post UVR ( $p=0.011$ at $24 \mathrm{~h}$ and 0.034 at $72 \mathrm{~h}$ ); whilst phototype I/II showed a consistent expression of eNOS principally in association with the dermal microvasculature for up to $72 \mathrm{~h}$, in phototype III/IV this was detected only at $4 \mathrm{~h}$ post UVR (Fig. 5I).

\subsection{Production of lipid mediators post UVR}

The production of lipid mediators was assessed in cutaneous blister fluid using a lipidomic (LC/ESI-MS/MS) assay. ${ }^{28,29}$ The n-6 PUFA arachidonic acid (AA)- derived prostaglandins $\mathrm{PGE}_{2}$ and $\mathrm{PGF}_{2 \alpha}$, and the dihomo-gamma linolenic acid (DGLA)-derived $\mathrm{PGE}_{1}$ were upregulated at $24 \mathrm{~h}$ post UVR in both phototype groups $(p<0.05)$ (Fig. 6A, B and C). However, in phototype I/II, $\mathrm{PGE}_{1}$ and $\mathrm{PGE}_{2}$ were still elevated at $72 \mathrm{~h}$ post UVR $(p=0.02$, $p=0.003$ respectively), whilst in skin type III/IV their production had returned to baseline levels (Fig. 6A and B).

The LOX product 12-HETE was upregulated at $24 \mathrm{~h}$ post UVR and its levels were still elevated at $72 \mathrm{~h}$ post UVR, with similar results in both phototype groups $(p=0.004)$ (Fig. 7A). However, 15-HETE was produced at higher levels in phototype $\mathrm{I} / \mathrm{II}$ at $72 \mathrm{~h}$ post $\mathrm{UVR}(p=0.0008)$ (Fig. $7 \mathrm{~B})$ and a strong positive correlation was shown between $\mathrm{PGE}_{2}$ and 15-HETE at this time point $(\mathrm{n}=18, r=0.805, p=0.0001)$. 15-HETE levels 


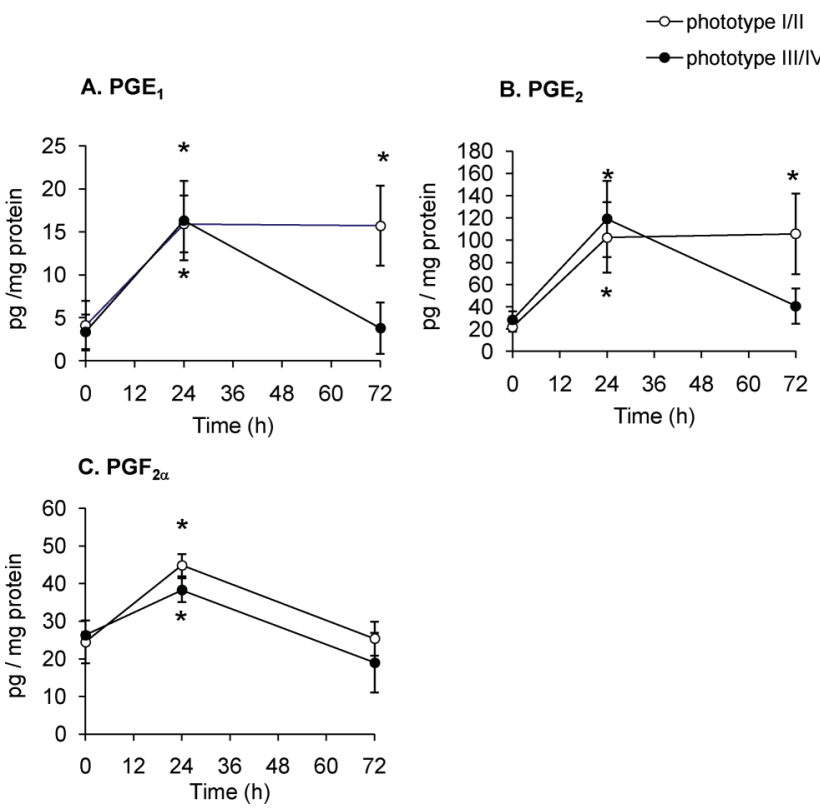

Fig. 6 Levels of prostaglandins $\mathrm{PGE}_{1}(\mathrm{~A}), \mathrm{PGE}_{2}(\mathrm{~B})$ and $\mathrm{PGF}_{2 \alpha}(\mathrm{C})$ in cutaneous blister fluid from unirradiated $(0 \mathrm{~h})$ and UVB irradiated (24 and $72 \mathrm{~h}$ ) human skin phototype I/II and III/IV, following 12 SED of UVR. Results are expressed as mean \pm SEM $(n=9$ subjects per skin phototype group). ${ }^{*} p<0.05$ comparing data to un-irradiated skin $(0 \mathrm{~h})$.

in phototype III/IV also appeared increased but the trend failed to reach statistical significance. A pattern similar to 15-HETE expression was also followed by the linoleic acid (LA)-derived 15LOX product 13-hydroxyoctadecadienoic acid (HODE), which was upregulated post UVR (Fig. 7E). Interestingly, levels of 13-HODE were significantly higher at 24 and $72 \mathrm{~h}$ post UVR compared with baseline in skin type I/II $(p=0.03)$, whilst there was no statistically significant rise in skin type III/IV (Fig. 7E). Finally, 11-HETE was elevated at $24 \mathrm{~h}$ and $72 \mathrm{~h}$ post UVR in both skin groups (Fig. 7C), whilst increased production of 8-HETE reached statistical significance at $72 \mathrm{~h}$ post UVR, in both phototypes ( $p=$ 0.02) (Fig. 7D).

\section{Discussion}

Bioactive lipid mediators contribute to the initiation, maintenance and also potentially the resolution of sunlight-induced cutaneous inflammation. The susceptibility of human skin to sunburn when exposed to solar UVR is reflected in the phototype classification. The easy burning phototype I/II is a strong risk factor for the longer term damage of photocarcinogenesis, ${ }^{1}$ and is also associated with photoageing and certain types of photosensitivity disorders. $^{30} \mathrm{~A}$ range of eicosanoids are upregulated in skin following UVR, ${ }^{10}$ and as well as expressing pro and/or antiinflammatory properties, they potentially contribute to other UVR-induced deleterious health effects through their additional properties including effects on cell proliferation, differentiation and immune function. ${ }^{13,31}$ We have recently reported that UVBinduced $\mathrm{PGE}_{2}$ production in epidermal melanocytes is positively correlated with low skin phototype of cell donor, suggesting that epidermal melanocytes may contribute to UVR-induced erythema. ${ }^{32}$
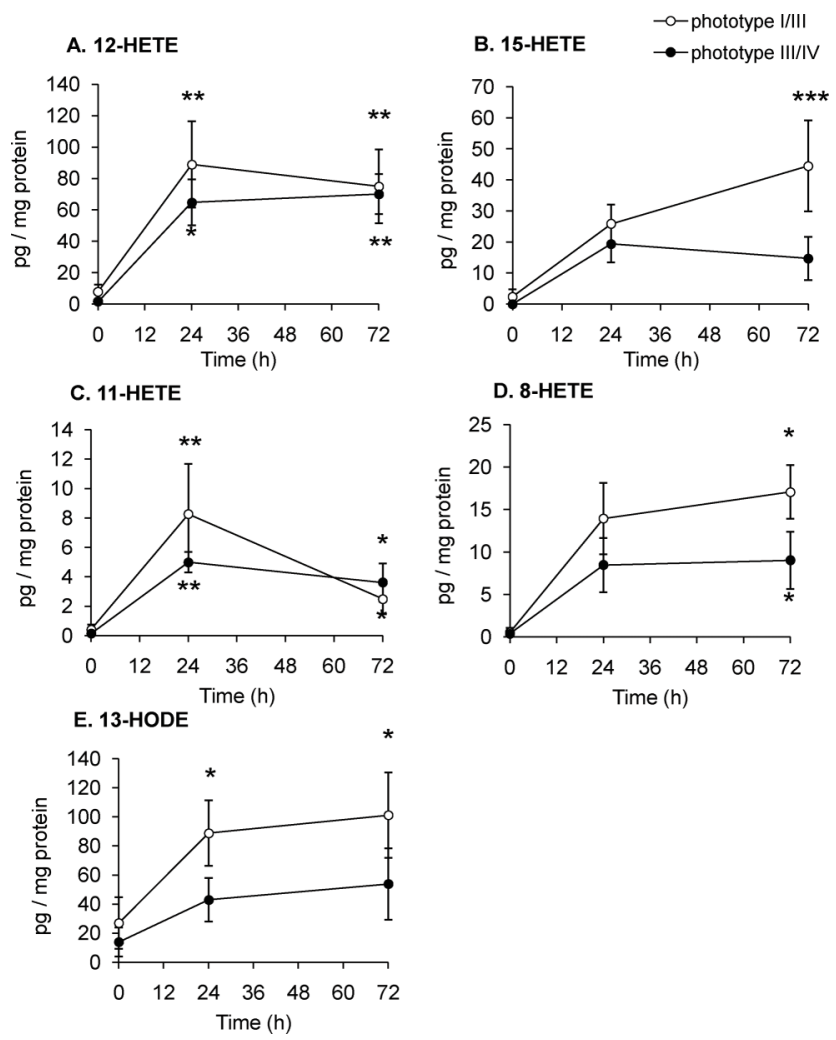$$
\text { . }
$$

Fig. 7 Levels of the hydroxy fatty acids 12-HETE (A), 15-HETE (B), 11-HETE (C), 8-HETE (D) and 13-HODE (E) in cutaneous blister fluid from unirradiated $(0 \mathrm{~h})$ and UVB irradiated (24 and $72 \mathrm{~h}$ ) human skin phototype I and IV, following 12 SED of UVR. Results are expressed as mean \pm SEM ( $\mathrm{n}=9$ subjects per phototype group). ${ }^{*} p<0.05,{ }^{* *} p<0.01$ and $* * * p<0.001$ comparing data to un-irradiated skin $(0 \mathrm{~h})$.

In this study, subjects were phototyped in the traditional manner by means of a standardised short interview focusing on personal experience of susceptibility to sunburn and suntan. ${ }^{2}$ Since these are subjective criteria, we confirmed a significant difference in sunburn threshold between phototype groups following direct phototesting, phototype I/II exhibiting a lower MED than phototype III/IV (Fig. 1). The response of each group to intense UVR was then studied over a period of $72 \mathrm{~h}$, following a single 12 SED exposure, as may be attained on unprotected skin sites during leisure activities in the summer months or on beach holidays. ${ }^{22,23}$ The same dose was applied to all subjects, rather than using a traditional MED-related dosing scheme, as people do not necessarily regulate or always have the ability to regulate, their sun exposure according to their propensity to burn. This also assisted our exploration of the relationships between anti-inflammatory and pro-inflammatory lipid mediators, and inflammatory outcomes.

Our erythemal data confirm that all subjects achieved a maximal erythema at $24 \mathrm{~h}$, in keeping with previous studies of the UVerythemal time course. ${ }^{5,30}$ Interestingly, while phototype I/II showed a greater inflammatory response at early time points, the groups equilibrated in their responses at the later time point of $72 \mathrm{~h}$ (Fig. 1C). Prostaglandins are important mediators of UV-induced cutaneous erythema, ${ }^{3-5,10}$ with recent evidence that $\mathrm{PGE}_{2}$ receptors EP2 and EP4 constitute a signalling system intimately involved in mediating the enhanced blood flow. ${ }^{33}$ Our findings confirm that 
$\mathrm{PGE}_{1}, \mathrm{PGE}_{2}$ and $\mathrm{PGF}_{2 \alpha}$ production was upregulated $24 \mathrm{~h}$ post UVR in both groups. However, the raised production of $\mathrm{PGE}_{1}$ and $\mathrm{PGE}_{2}$ was considerably more prolonged in phototype $\mathrm{I} / \mathrm{II}$, i.e. to $72 \mathrm{~h}$ (Fig. 6), reflecting the overall greater inflammatory response as anticipated in this group. Immunohistochemical analysis confirmed significant UVR-upregulation of the inducible COX-2, in the epidermis and dermis of phototype I/II; this isoform of COX produces high concentrations of prostaglandins and can therefore explain the observed prolonged production of $\mathrm{PGE}_{1}$ and $\mathrm{PGE}_{2}$. Conversely, phototype III/IV was characterised by upregulation of the housekeeping isoform COX-1 in epidermis and dermis (Fig. 3), believed to contribute lower levels of prostaglandins.

Nitric oxide synthase expression was examined in addition to lipid metabolising enzymes, as NO may also contribute significantly to acute UVR-induced erythema production. ${ }^{4,5}$ While the exact role of each NOS isoform in the UV response is unresolve $\mathrm{d}^{20}$ there is evidence that UVR-induced NO may be largely attributable to dermal rather than epidermal production, with high levels found in dermal microdialysate, rather than in suction blister fluid, of human skin following UVR. ${ }^{5}$ Our analysis of the three NOS isoforms revealed that dermal expression of the endothelial-type, i.e. eNOS, was almost exclusively in phototype I/II (Fig. 5I), and a similar pattern of higher dermal expression was noted for the neuronal type, i.e. nNOS. This upregulation of two NOS isoforms in the dermis of skin phototype I/II, could lead to production of higher concentrations of NO by cells close to or within dermal blood vessels, thus enhancing vasodilatation ${ }^{34}$ and potentially contributing to the early appearance and trend for higher levels of erythema shown in phototype I/II (Fig. 1). In contrast, the inducible form, i.e. iNOS, was significantly upregulated only in the epidermis of skin phototype III/IV and not in type I/II (Fig. 5D-E); this finding may speculatively be linked to the melanogenic properties of NO and could facilitate the ability of phototype III/IV to tan. ${ }^{35}$

Neutrophil and lymphocyte influx post UVR was seen in both phototypes, although with notable differences. Phototype I/II was characterised by a significant infiltration of neutrophils at $24 \mathrm{~h}$ post UVR (Fig. 2A) whilst phototype III/IV instead showed a conspicuous infiltration of CD3+ lymphocytes at this early time point (Fig. 2B). Neutrophils are potent pro-inflammatory cells, releasing reactive oxygen species and chemokines that further augment the leucocytic infiltration, while HETE are potent leucocyte chemoattractants ${ }^{36,37}$ that may have contributed to the dermal neutrophil influx. Indeed, our findings indicate a trend for higher expression of 12-HETE, 11-HETE and 8HETE in phototype I/II than III/IV, accompanied by significant upregulation of cutaneous 12-LOX expression, all consistent with the greater inflammatory status of this group (Fig. 4, 7).

Interestingly, the anti-inflammatory 15 -HETE, reported to inhibit 12-LOX activity in human platelets, ${ }^{38} \mathrm{HaCaT}$ keratinocytes ${ }^{14}$ and skin sections, ${ }^{15}$ appears significantly raised at $72 \mathrm{~h}$ post UVR in phototype I/II. Furthermore, $\mathrm{PGE}_{2}$, that has been shown to induce production of 15-HETE in clinical and experimental exudates, ${ }^{17}$ was also elevated in this phototype at $72 \mathrm{~h}$ post UVR. Whilst the pattern of 15-LOX expression appeared similar for both phototype groups, the significant elevation of AA-derived 15-HETE and LA-derived 13-HODE in phototype I/II (Fig. 7B and 7E) indicated increased 15-LOX activity in this subject group. The rise in 15-HETE at $72 \mathrm{~h}$ might therefore explain the reduction in neutrophil numbers at this later time point in phototype I/II, potentially through inhibition or attenuation of the activity of 12-LOX-derived chemoattractant 12-HETE. Moreover, both 15HETE and 13-HODE are anti-inflammatory endogenous ligands of PPAR $\gamma^{39,40}$ whilst 13-HODE can be incorporated into substituted diacylglycerols with anti-inflammatory/anti-proliferative effects ${ }^{41}$ and 15-HETE is the biochemical precursor of lipoxins. ${ }^{16}$ Consequently, the anti-inflammatory profile of these 15-LOXderived lipids found upregulated in phototype I/II, suggests they may play role in modulating the degree of inflammation.

The earlier accumulation of CD3+ lymphocytes in phototype III/IV compared with I/II is an intriguing finding warranting further investigation. While CD3+/CD4+ lymphocytic infiltration has been reported to occur later in the sunburn reaction relative to neutrophil influx, ${ }^{7,9,10}$ we are unaware of previous studies dissecting out differences in phototype responses, or under greater or lesser UV-induced inflammatory states. Further work is thus indicated to characterise these cells and examine their specific role in the modulation of the inflammation/immune response to UVR. In contrast to the observed differences in inflammatory characteristics between phototypes during the early phase of the sunburn response, i.e. 4-24 h post-UVR, both the histological (leukocytic infiltrate) and clinical (erythema) evidence of inflammation were equivalent between phototypes $\mathrm{I} / \mathrm{II}$ and III/IV in the late phase, i.e. at $72 \mathrm{~h}$. This, however, may result from a balanced higher expression of both pro- and antiinflammatory eicosanoids, including pro-inflammatory $\mathrm{PGE}_{2}$ and anti-inflammatory 15-HETE, in phototype I/II. Furthermore, detailed examination of the EP receptors in skin types I/II and III/IV may potentially assist elucidation of the mechanism of the differing roles of this potent mediator during the UV response.

In conclusion, our data indicate that human skin predisposed to sunburn shows greater inflammation in the early phase (4$24 \mathrm{~h}$ ) following UVR, with contributions by 12-LOX, COX2 and eNOS, than those that are more resistant to sunburn, when exposed to the same intense UVR challenge. While in the later phase $(72 \mathrm{~h})$ the clinical and histological inflammation is similar in the two groups, there is higher expression of both proinflammatory $\left(\mathrm{PGE}_{1}, \mathrm{PGE}_{2}\right)$ and anti-inflammatory (15-HETE) eicosanoids in those with a tendency to sunburn. Although some of these differences are small, COX-2, 12-LOX and PG have tumour promoting and/or immunomodulatory properties s $^{1,42-44}$ which may become increasingly relevant with repeated UVR exposures. Due to the multiple sampling procedures in this study, the subgroups for histological analysis are necessarily of limited size and further differences might be found with a larger sample; further studies should also aim to directly demonstrate the anti-inflammatory role of 15-HETE under these experimental conditions. This study highlights potential lipid targets for pharmacological intervention, which may help reduce the elevated risk of UVR-related adverse health events in phototypes I/II.

\section{List of abbreviations}

$\begin{array}{ll}\text { AA } & \text { arachidonic acid } \\ \text { COX } & \text { cyclooxygenase } \\ \text { CYP } & \text { cytochrome P450 oxygenase } \\ \text { ESI } & \text { electrospray ionisation } \\ \text { HETE } & \text { hydroxyeicosatetraenoic acid }\end{array}$




$\begin{array}{ll}\text { HODE } & \text { hydroxyoctadecadienoic acid } \\ \text { LC-MS/MS } & \begin{array}{l}\text { liquid chromatography coupled to tandem mass } \\ \text { spectrometry }\end{array} \\ \text { LOX } & \text { lipoxygenase } \\ \text { MED } & \text { minimal erythema dose } \\ \text { MRM } & \text { multiple reaction monitoring } \\ \text { NO } & \text { nitric oxide } \\ \text { NOS } & \text { nitric oxide synthase } \\ \text { PG } & \text { prostaglandins } \\ \text { PUFA } & \text { polyunsaturated fatty acids } \\ \text { SED } & \text { standard erythema dose } \\ \text { UVR } & \text { ultraviolet radiation }\end{array}$

\section{Acknowledgements}

We acknowledge the Wellcome Trust (project number WT077714) for funding this research. The group of LER is supported by the Manchester Academic Health Sciences Centre (MAHSC) and the NIHR Manchester Biomedical Research Centre. The authors thank Dr R. Watson for useful advice and Andrew Healey, University of Bradford Analytical Centre for technical support.

\section{References}

1 Solar and Ultraviolet Radiations: Monograph on the Evaluation of Carcinogenic Risks to Humans - IARC Monographs v. 55, IARC Monographs, 1992.

2 T. B. Fitzpatrick, The validity and practicality of sun-reactive skin types I through VI, Arch. Dermatol., 1988, 124, 869-871.

3 A. K. Black, M. W. Greaves, C. N. Hensby and N. A. Plummer, Increased prostaglandins E2 and F2alpha in human skin at 6 and 24 h after ultraviolet B irradiation (290-320 nm), Br J Clin Pharmacol, 1978, 5, 431-436.

4 J. B. Warren, Nitric oxide and human skin blood flow responses to acetylcholine and ultraviolet light, FASEB J, 1994, 8, 247-251.

5 L. E. Rhodes, G. Belgi, R. Parslew, L. McLoughlin, G. F. Clough and P. S. Friedmann, Ultraviolet-B-induced erythema is mediated by nitric oxide and prostaglandin E2 in combination, J. Invest. Dermatol., 2001, $\mathbf{1 1 7}, 880-885$.

6 I. Strickland, L. E. Rhodes, B. F. Flanagan and P. S. Friedmann, TNFalpha and IL-8 are upregulated in the epidermis of normal human skin after UVB exposure: correlation with neutrophil accumulation and Eselectin expression, J. Invest. Dermatol., 1997, 108, 763-768.

7 M. B. Teunissen, G. Piskin, S. di Nuzzo, R. M. Sylva-Steenland, M. A de Rie and J. D. Bos, Ultraviolet B radiation induces a transient appearance of IL-4+ neutrophils, which support the development of Th2 responses, J Immunol, 2002, 168, 3732-3739.

8 J. L. Hawk, G. M. Murphy and C. A. Holden, The presence of neutrophils in human cutaneous ultraviolet-B inflammation, $B r . J$. Dermatol., 1988, 118, 27-30.

9 S. Di Nuzzo, R. M. Sylva-Steenland, M. A. de Rie, P. K. Das, J. D. Bos and M. B. Teunissen, UVB radiation preferentially induces recruitment of memory CD4+ T cells in normal human skin: long-term effect after a single exposure, J. Invest. Dermatol., 1998, 110, 978-981.

10 L. E. Rhodes, K. Gledhill, M. Masoodi, A. K. Haylett, M. Brownrigg, A. J. Thody, D. J. Tobin and A. Nicolaou, The sunburn response in human skin is characterized by sequential eicosanoid profiles that may mediate its early and late phases, FASEB J., 2009, 23, 3947-3956.

11 V. A. Ziboh, Prostaglandins, leukotrienes, and hydroxy fatty acids in epidermis, Semin Dermatol, 1992, 11, 114-120.

12 J. H. Capdevila, J. R. Falck and R. W. Estabrook, Cytochrome P450 and the arachidonate cascade, FASEB J, 1992, 6, 731-736.

13 A. Nicolaou, S. M. Pilkington and L. E. Rhodes, Ultraviolet-radiation induced skin inflammation: dissecting the role of bioactive lipids, Chem. Phys. Lipids, 2011, 164, 535-543.

14 H. Yoo, B. Jeon, M. S. Jeon, H. Lee and T. Y. Kim, Reciprocal regulation of 12- and 15-lipoxygenases by UV-irradiation in human keratinocytes, FEBS Lett., 2008, 582, 3249-3253.
15 K. Kragballe, G. Pinnamaneni, L. Desjarlais, E. A. Duell and J. J. Voorhees, J. Invest. Dermatol., 1986, 87, 494 498.

16 A. Ryan and C. Godson, Lipoxins: regulators of resolution, Curr. Opin. Pharmacol., 2010, 10, 166-172.

17 B. D. Levy, C. B. Clish, B. Schmidt, K. Gronert and C. N. Serhan, Lipid mediator class switching during acute inflammation: signals in resolution, Nat. Immunol., 2001, 2, 612-619.

18 M. Di Rosa, A. Ialenti, A. Ianaro and L. Sautebin, Interaction between nitric oxide and cyclooxygenase pathways, Prostaglandins, Leukotrienes Essent. Fatty Acids, 1996, 54, 229-238.

19 K. S. Chun, J. K. Akunda and R. Langenbach, Cyclooxygenase2 inhibits UVB-induced apoptosis in mouse skin by activating the prostaglandin E2 receptors, EP2 and EP4, Cancer Res., 2007, 67, 20152021.

20 M. M. Cals-Grierson and A. D. Ormerod, Nitric oxide function in the skin, Nitric Oxide, 2004, 10, 179-193.

21 H. M. Sowden, K. M. Naseem and D. J. Tobin, Differential expression of nitric oxide synthases in human scalp epidermal and hair follicle pigmentary units: implications for regulation of melanogenesis, $B r . J$. Dermatol., 2005, 153, 301-309.

22 M. A. Serrano, J. Canada and J. C. Moreno, Erythemal ultraviolet exposure of cyclists in Valencia, Spain, Photochem. Photobiol., 2010, 86, 716-721.

23 D. L. O'Riordan, A. D. Steffen, K. B. Lunde and P. Gies, A day at the beach while on tropical vacation: sun protection practices in a highrisk setting for UV radiation exposure, Arch. Dermatol., 2008, 144, $1449-1455$.

24 B. L. Diffey, C. T. Jansen, F. Urbach and H. C. Wulf, The standard erythema dose: a new photobiological concept, Photodermatol., Photoimmunol. Photomed., 1997, 13, 64-66.

25 A. R. Webb, R. Kift, J. L. Berry and L. E. Rhodes, The vitamin D debate: translating controlled experiments into reality for human sun exposure times, Photochem. Photobiol., 2011, 87, 741-745.

26 B. L. Diffey and P. M. Farr, Quantitative aspects of ultraviolet erythema, Clin. Phys. Physiol. Meas., 1991, 12, 311-325.

27 L. E. Rhodes, B. H. Durham, W. D. Fraser and P. S. Friedmann, Dietary fish oil reduces basal and ultraviolet B-generated PGE2 levels in skin and increases the threshold to provocation of polymorphic light eruption, J. Invest. Dermatol., 1995, 105, 532-535.

28 M. Masoodi and A. Nicolaou, Lipidomic analysis of twenty-seven prostanoids and isoprostanes by liquid chromatography/electrospray tandem mass spectrometry, Rapid Commun. Mass Spectrom., 2006, 20, 3023-3029.

29 M. Masoodi, A. A. Mir, N. A. Petasis, C. N. Serhan and A. Nicolaou, Simultaneous lipidomic analysis of three families of bioactive lipid mediators leukotrienes, resolvins, protectins and related hydroxy-fatty acids by liquid chromatography/electrospray ionisation tandem mass spectrometry, Rapid Commun. Mass Spectrom., 2008, 22, 75-83.

30 L. E. Rhodes, M. Bock, A. S. Janssens, T. C. Ling, L. Anastasopoulou, C. Antoniou, F. Aubin, T. Bruckner, B. Faivre, N. K. Gibbs, C. Jansen, S. Pavel, A. J. Stratigos, F. R. de Gruijl and T. L. Diepgen, Polymorphic light eruption occurs in $18 \%$ of Europeans and does not show higher prevalence with increasing latitude: multicenter survey of 6895 individuals residing from the Mediterranean to Scandinavia, $J$. Invest. Dermatol., 2010, 130, 626-628.

31 S. M. Pilkington, R. E. Watson, A. Nicolaou and L. E. Rhodes, Omega3 polyunsaturated fatty acids: photoprotective macronutrients, Exp. Dermatol., 2011, 20, 537-543.

32 K. Gledhill, L. E. Rhodes, M. Brownrigg, A. K. Haylett, M. Masoodi, A. J. Thody, A. Nicolaou and D. J. Tobin, Prostaglandin-E2 is produced by adult human epidermal melanocytes in response to UVB in a melanogenesis-independent manner, Pigm. Cell Melanoma Res., 2010, 23, $394-403$.

33 K. Kabashima, M. Nagamachi, T. Honda, C. Nishigori, Y. Miyachi, Y. Tokura and S. Narumiya, Prostaglandin E2 is required for ultraviolet B-induced skin inflammation via EP2 and EP4 receptors, Lab. Invest., 2007, 87, 49-55.

34 D. L. Kellogg, Jr., J. L. Zhao and Y. Wu, Neuronal nitric oxide synthase control mechanisms in the cutaneous vasculature of humans in vivo, $J$. Physiol., 2008, 586, 847-857.

35 C. Romero-Graillet, E. Aberdam, M. Clement, J. P. Ortonne and R. Ballotti, Nitric oxide produced by ultraviolet-irradiated keratinocytes stimulates melanogenesis, J. Clin. Invest., 1997, 99, 635-642.

36 K. B. Bacon, R. D. Camp, F. M. Cunningham and P. M. Woollard, Contrasting in vitro lymphocyte chemotactic activity of the hydroxyl 
enantiomers of 12-hydroxy-5,8,10,14-eicosatetraenoic acid, Br J Pharmacol, 1988, 95, 966-974.

37 E. J. Goetzl, A. R. Brash, A. I. Tauber, J. A. Oates and W. C. Hubbard, Modulation of human neutrophil function by monohydroxy-eicosatetraenoic acids, Immunology, 1980, 39, 491501 .

38 M. Fletcher-Cieutat, J. Y. Vanderhoek, R. W. Bryant and J. M. Bailey, Aspirin enhances the sensitivity of human platelet 12-lipoxygenase to inhibition by 15-HETE, an endogenous regulator, Prostaglandins, Leukotrienes Med., 1985, 18, 255-259.

39 T. Itoh, L. Fairall, K. Amin, Y. Inaba, A. Szanto, B. L. Balint, L. Nagy, K. Yamamoto and J. W. Schwabe, Structural basis for the activation of PPARgamma by oxidized fatty acids, Nat. Struct. Mol. Biol., 2008, 15, 924-931.

40 R. Limor, O. Sharon, E. Knoll, A. Many, G. Weisinger and N. Stern, Lipoxygenase-derived metabolites are regulators of peroxisome proliferator-activated receptor gamma-2 expression in human vascular smooth muscle cells, Am. J. Hypertens., 2008, 21, 219-223.

41 V. A. Ziboh, C. C. Miller and Y. Cho, Significance of lipoxygenasederived monohydroxy fatty acids in cutaneous biology, Prostaglandins Other Lipid Mediators, 2000, 63, 3-13.

42 S. Y. Buckman, A. Gresham, P. Hale, G. Hruza, J. Anast, J. Masferrer and A. P. Pentland, COX-2 expression is induced by UVB exposure in human skin: implications for the development of skin cancer, Carcinogenesis, 1998, 19, 723-729.

43 Y. Fujimoto, H. Iwagaki, M. Ozaki, T. Ogino, H. Murata, D. S. Sun, H. Sadamori, H. K. Takahashi, N. Tanaka and T. Yagi, Involvement of prostaglandin receptors (EPR2-4) in in vivo immunosuppression of PGE2 in rat skin transplant model, Int. Immunopharmacol., 2005, 5, 1131-1139.

44 D. Wang and R. N. Dubois, Prostaglandins and cancer, Gut, 2006, 55, $115-122$. 